\title{
BUCHBESPRECHUNGEN
}

Flügel-Martinsen, Oliver: Grundfragen politischer Philosophie. Eine Untersuchung der Diskurse über das Politische.

(Rainer Miebe)........................... 353

Hirsch, Michael: Die zwei Seiten der Entpolitisierung. Zur politischen Theorie der Gegenwart. (Holger Zapf)... 354

Munier, Gerald: Thomas Morus. Urvater des Kommunismus und katholischer Heiliger und Ribhegge, Wilhelm: Erasmus von Rotterdam.

(Thomas Schölderle)....................... 355

Thein, Martin: Wettlauf mit dem Zeitgeist - Der Neonazismus im Wandel. Eine Feldstudie. (Armin Pfabl-Trangbber) 359

Oliver FLÜGEL-MARTINSEN: Grundfragen politischer Philosophie. Eine Untersuchung der Diskurse über das Politische, Nomos Verlag Baden-Baden 2008, 277 S., kart., 19,90€

Wenn Denker wie Sloterdijk das Politische durch die Philosophie auflösen wollen, um mit thymotischer Energie eine »Revolution der gebenden Hand « (FAZ vom 10.6.09) auszulösen, dann wird einem klar, wie nötig instruktive Betrachtungen zur politischen Philosophie geworden sind, schon allein aus Gründen der Unsinnsreduktion.

Flügel-Martinsens Buch ist vergleichsweise instruktiv. Vier Diskurse werden vorgestellt: Begründen, Befestigen, Begrenzen, Befragen. Sie stellen gewissermaßen einen ideengeschichtlichen Verlauf dar, der die Reflexionen über das Politische vor dem Hintergrund normativer Welt- und Menschenbilder kennzeichnet danach, was in diesen Reflexionen geschieht. Aristotelische, Kantische und Hegelianische Diskurse begründen das Gemeinwesen und realisieren Freiheit auf jeweils unterschiedliche Weise durch Institutionen, die das Regierungshandeln in allen Politikfeldern legitimieren soll.

Befestigen ist notwendig, weil die menschliche Natur mit ihren Bestrebungen nach Macht und Gewalt auch immer eine Gefahr des begründeten Gemeinwesens darstellt. Ordnung und Sicherheit treten in den Vordergrund und damit die auf Machiavelli und Hobbes zurückgehenden Diskurse. Hier stellt sich die Frage, wie weit der Staat in die Freiheit des Einzelnen eingreifen darf, so dass Probleme der Begrenzung entstehen. Diese Probleme werden vom Autor durch Heranziehung der Konzeptionen vor allem von Locke, Rawls und Nozick diskutiert.

Abschließend führt Flügel-Martinsen den Diskurs des Befragens ein, der im wesentlichen ein Nietzscheanischer ist und die Verunsicherung allen Denkens zum Gegenstand hat.

Flügel-Martinsen beherrscht sein Metier so, wie es in der Sozial- und Geisteswissenschaft weitgehend gefordert wird. Nicht Tatsachen und detaillierte Einzeluntersuchungen stehen an erster Stelle, sondern der große Überblick, hier der souveräne Umgang mit den ideengeschichtlichen Strömungen. Der hermeneutische Wahrheitsanspruch, den der Autor vertritt, ergibt sich dadurch, dass die eloquente Art, wie dieser Anspruch auftritt, schon allein die Geltung von Wahrheit zu belegen scheint.

Dazu gehört die Aussage (26): »Obzwar die Regierungsform der Demokratie im Verlauf der politischen Ideengeschichte eine allseits ungeliebte Konzeption darstellt, die geradezu gar keinen Fürsprecher mobilisieren kann und - nicht allein - von Aristoteles, Kant und Hegel unisono als denkbar schlechtester Zustand apostrophiert wird, steht sie mittlerweile im Zentrum der theoretischen und praktischen politischen Diskurse.« Dass diese kategorische Bewertung abwegig ist, ergeben flüchtige Blicke auf die englischen Theoretiker des 18. und 19. Jahrhunderts und vor allem auf Kant, der demokratische Elemente in seiner Konzeption von der Republik sehr wohl hervorhebt.

Flügel-Martinsen referiert hingegen gut nachvollziehbar und einleuchtend den Freiheitsbegriff von Hannah Arendt, durch welchen der Zusam- 
menhang von Gründen und Bewabren deutlich wird. Arendt liegt es fern, Politik durch Philosophie aufzulösen. Freiheit erfahren die Menschen im gemeinsamen Handeln, aus dem heraus überhaupt erst Weltbezüge entstehen.

Was der Leser in der Studie vermisst, ist die Einbeziehung von Religion bzw. Offenbarung im politischen Denken. Flügel-Martinsens Resümee, »das Politische unterhält Beziehungen zum Normativen « (261), wirkt im Vergleich zu den vielen komplizierten Reflexionen des Autors sehr karg, und man hätte sich gewünscht, wenn das Wechselspiel von Offenheit und Geschlossenheit der Diskurse zu einer dichteren Quintessenz geführt hätte.

Außerdem ist die Zuspitzung der Nietzscheanischen Diskurse auf die französische Philosophie der Gegenwart ziemlich einseitig. Nietzsche war kein vornehmlich politischer Denker. Hier hätte der deutsche bzw. internationale Forschungsstand stärker berücksichtigt werden sollen. Auch der Einfluss Nietzsches auf die normativen Klassiker der Politikwissenschaft, Eric Voegelin und vor allem Leo Strauss, gehört mit zu den Grundlagen politischer Philosophie.

Wer indes wissen will, wie politische Philosophie heute betrieben wird, hält eine lesenswerte Schrift in den Händen.

\section{Rainer Miebe}

\section{Michael HIRSCH: Die zwei Seiten der Entpoliti- sierung. Zur politischen Theorie der Gegenwart, Stuttgart: Franz Steiner Verlag 2007, 214 S., brosch., 36,00 EUR.}

Der demokratische Rechtsstaat ist am Ende. Er ist am Ende, bevor es ihn jemals gegeben hat. Das abstrakt-jungfräuliche normative Konzept einer erst noch einzulösenden Volkssouveränität, das ihn eigentlich garantieren sollte, wurde und wird von perversen Gegenaufklärern geschändet. Das geschieht auf zweierlei Weise: Entweder wird darauf verwiesen, dass der Staat als Zurechnungsort von im Volkswillen begründeter Normativität längst verlorengegangen ist und nur noch als semantische Chimäre fortlebt, was sich empirisch in der Tendenz spiegelt, dass sich Staatshandeln mehr und mehr in einer (zumindest scheinbar) kooperativen Subpolitik verliert, die gesellschaftliche Kräfteverhältnisse reproduziert. Oder aber die Volkssouve- ränität wird verdreht in eine nur außerstaatlich mögliche und damit nicht normierbare Eruption gesellschaftlicher Kräfte, die sich als pouvoir constituant gegen den Staat als pouvoir constitué wenden. Aus dieser doppelten Frontstellung kann das kantische Aufklärungsdenken jedoch befreit werden, wenn man sich bewusst macht, dass die Gleichsetzung normativer und diagnostischer Konzepte ein Kategorienfehler ist. Das ist, wenn man so will, die Erzählung, die Michael Hirschs Buch - hervorgegangen aus einer Dissertation bei Ingeborg Maus - zugrunde liegt.

Es handelt sich dabei um eine für einen »Normativisten « mutige Auseinandersetzung mit etwa zwanzig neueren Theorieentwürfen aus einer an Kant orientierten Perspektive. Die Diagnose ist dabei eine doppelte: Die untersuchten Theorien ziehen sich angesichts der Komplexität von Politik und Gesellschaft entweder auf eine technokratische oder auf eine ontologische bzw. ästhetische Position zurück. Dadurch kommt es Hirsch zufolge zu einer Entrechtlichung von Staat und Gesellschaft, insofern nicht nur die Verwirklichung demokratischer Gleichheit als Projekt aufgegeben wird, sondern überhaupt das Regulativ einer funktionierenden Demokratie wegbricht: Die eher linke "politische Ontologie - von Rancière bis Badiou - setzt dem als Demokratie gescheiterten Staat eine symbolische Gemeinschaft entgegen, die, von der Idee der Volkssouveränität abgekoppelt, nicht mehr darauf angelegt ist, im Rahmen demokratischer Verfahren Legitimität zu erzeugen. Die "politische Technokratie« auf der anderen Seite tendiert dazu, Staatshandeln von der legislativen Kontrolle weg zunehmend in den Hoheitsbereich der Verwaltung zu verschieben, was dazu führt, dass der Staat, ohne sich weiterhin des Steuerungsmediums demokratisch legitimierten Rechts zu bedienen, zum Akteur auf subpolitischer Ebene wird. Dies sind die "zwei Seiten der Entpolitisierung «. Mit dieser Analyse wird zugleich aufgezeigt, wie sehr scheinbar linke Theorien konservativen und fortschrittsfeindlichen Positionen verfallen sind. Diesem Verdikt unterliegen dann auch nicht nur die autoritären politischen Theorien der Einheit von Hegel und Schmitt, sondern auch die der Differenz von Lyotard über Deleuze bis zu Luhmann - Entpolitisierung, soweit das Auge reicht. Das Korrektiv gegenüber diesen normativen Sackgassen ist die starke Annahme der 
Möglichkeit eines an Volkssouveränität rückgebundenen staatlichen Rechts.

Auf den einen oder anderen mag es zwar ermüdend wirken, wie Hirsch die beiden Arten von Entpolitisierung an einem Spektrum von avancierten politischen Theorien der Gegenwart durchdekliniert und herausarbeitet, wie sie sich in ihrer Tendenz zur technokratischen oder ontologischen Entpolitisierung gleichen - doch hat dieser Aufweis selbst zeitdiagnostische Qualität, so dass man sich davon durchaus beunruhigen lassen kann. Methodisch bemerkenswert ist dabei insbesondere, dass im Anschluss an Luhmann immer wieder auf die (ideologische) Funktion von Theoriesemantiken verwiesen wird - denn das ist in der Politischen Theorie gewiss ein Bereich, der mittelfristig an Bedeutung gewinnen wird. Insofern ist es bedauerlich, dass auf die methodische Entwicklung der Untersuchung dieser Semantiken nicht eingegangen wird - wenn auch durch die Darstellung einmal mehr offensichtlich wird, dass hier einiges an Material vorliegt, das eine eingehende, auf diese Weise methodisch fundierte Untersuchung verdient hätte. Einstweilen kann man Hirschs eigene Entpolitisierungssemantik goutieren als den Versuch, dem sozialwissenschaftlichen Phantasma überbordender und nicht steuerbarer Komplexität, das der Entpolitisierung zugrunde liegt, ein wohlartikuliertes Unbehagen entgegenzuhalten, das der Aufklärung verpflichtet ist. Also: Endlich wieder eine Utopie, die nicht durch ihre Radikalität und ihren Willen zur (theoretischen) Gewalt besticht! Oder, wie es der Autor vorsichtig formuliert: "Es könnte sein, $[\ldots]$ dass das normativ Richtige nicht unbedingt immer neu und interessant ist. «In einer auf Innovationszwang abstellenden Wissenschaftskultur ist diese Aussage ein Ausdruck von Integrität, im Hinblick auf die Untersuchung weist sie mit vernünftiger Nüchternheit die Grenzen derselben auf. Kann man mehr verlangen?

Holger Zapf
Gerald MUNIER: Thomas Morus. Urvater des Kommunismus und katholischer Heiliger. Hamburg: VSA-Verlag 2008, 340 S., brosch., 22,80 EUR und Wilhelm RIBHEGGE: Erasmus von Rotterdam. Darmstadt: Wissenschaftliche Buchgesellschaft 2010, 278 S., geb., 29,90 EUR.

Den Zeitgenossen schienen beide unzertrennlich, manchen galten sie sogar als »Zwillingspaar « (vgl. Hubertus Schulte Herbrüggen, Einleitung, in: Thomas-Morus-Werke, Bd. 5: Briefe der Freundschaft mit Erasmus, München 1985, S. 36). Am 31. August 1535, knapp zwei Monate nachdem Thomas Morus auf dem Towerhügel enthauptet worden war, schrieb der zutiefst betrübte Erasmus: »Mit Morus' Tod scheine ich selbst gestorben, war meine Seele doch zweigeteilt « (Erasmus von Rotterdam: Opus Epistolarum Des. Erasmi Roterodami, 12 Bde., hrsg. v. Percy S. Allen u.a., Oxford 1906-1958, hier Bd. XI, 3049, Z. 163 f.) Auch Morus beendete bereits im Jahr 1529 einen Brief an Erasmus mit den Worten: "Leb wohl, mein lieber Erasmus, du mehr als die Hälfte meiner Seele « (Morus an Erasmus, 28. Oktober 1529, aaO. (FN 1), Nr. 47, S. 290). Die tiefe Freundschaft der beiden großen Humanisten ist nicht nur um ihrer selbst willen bemerkenswert. Immer wieder liefert die Beziehung auch allerlei Klärendes für das Verständnis ihrer jeweiligen Werke, nicht zuletzt für Morus' berühmte Utopia (1516), Erasmus' Fürstenspiegel Erziehung des christlichen Fürsten (1516) oder dessen Geniestreiche im Lob der Torbeit (1511).

$\mathrm{Zu}$ den beiden großen Figuren der Renaissance sind nun zwei neue, allerdings höchst unterschiedliche Biografien erschienen. Gerald Muniers Buch Thomas Morus. Urvater des Kommunismus und katholischer Heiliger kam im März 2008 im Hamburger VSA-Verlag auf den Markt; Wilhelm Ribhegges Erasmus von Rotterdam erschien im Januar 2010 bei der Wissenschaftlichen Buchgesellschaft. In beiden Lebensbeschreibungen ist das innige Freundschaftsverhältnis zentrales Thema. Munier widmet der Beziehung unter der Überschrift »Der Weggefährte Erasmus von Rotterdam « ein eigenes Kapitel. Und in Ribhegges Erasmus-Buch ist Morus eigentlich unaufhörlich präsent; der mit $\mathrm{Ab}$ stand umfangreichste Eintrag im Namensregister ist "Morus « vorbehalten. Damit haben sich die Gemeinsamkeiten beider Bücher aber bereits weitgehend erschöpft. Während Ribhegge eine nüch- 
terne Bestandsaufnahme der biografischen Forschung liefert und die Lebensgeschichte Erasmus' sachlich abwägend nachzeichnet, verfolgt Munier eine Mission: Pointiert, zuweilen polemisch, versucht er seine These zu untermauern, die lautet: Morus sei entgegen der hagiografischen Tradition sowohl katholischer wie sozialistischer Biografen in Wahrheit eine ausgesprochen widersprüchliche, ja höchst angreifbare Person gewesen.

Morus (1478-1535) ist fraglos eine schillernde Figur. Er ist nicht nur Autor der Utopia (1516) und damit zugleich Namensgeber einer neuzeitlichen Gattungs- und Denktradition; eine steile politische Laufbahn führte ihn zudem bis in das höchste Amt unter dem König: Von 1529 bis 1532 war Morus englischer Lordkanzler. Seinen Weltruhm verdankt er aber hauptsächlich seiner Weigerung, den Eid auf die neue, von Rom losgerissene Anglikanische Kirche Heinrichs VIII. zu leisten - eine Weigerung, die ihm das Leben kostete. Für die Standhaftigkeit sprach ihn die katholische Kirche 1935 heilig. Der Märtyrer diente aber nicht nur der katholischen Kirche als leuchtendes Vorbild; auch die sozialistische Rezeption entdeckte Morus als einen ihrer Vordenker und hob ihn als ersten Verkünder eines kommunistischen Gesellschaftsmodells auf den Schild. Unabhängig von ideologischen Interessen nötigt das gelassene und heitere Sterben des Morus aber bis heute jedem einfachen Betrachter Respekt ab. Noch auf dem Weg zum Schafott soll er scherzend zum Gouverneur des Towers gesagt haben: »Helft mir bitte beim Hinaufsteigen, Master Kommandant, für mein Herunterkommen lasst mich selber sorgen « (vgl. William Roper, Das Leben des Thomas Morus, Heidelberg 1986, S. 87). Außerdem habe er seinen langen weißen Bart beim Niederknien augenzwinkernd zur Seite geschoben und in Richtung Henker gemeint: »Mein Bart zumindest ist des Hochverrats nicht schuldig. Nimm deinen Mut zusammen, mein Hals ist sehr kurz, darum sieh dich um deiner Ehre willen vor, dass du nicht verkehrt schlägst.«

In der Tat eignet sich Morus' Leben und Sterben daher als Vorlage für glorifizierende Porträts, von denen es wahrlich genügend gibt. Demgegenüber zeichnet Muniers Buch zumindest auf den ersten Blick eine wohltuend distanzierte Perspektive aus, die vorgibt, den wirklichen Menschen hinter den Geschichten und Legenden zu ergründen. Doch schon bald stellt sich der Eindruck ein, dass die sensationslüsterne Demontage das einzige Ziel der Darstellung ist. Wenn die bisherige Morus-Forschung nicht völlig irrt, dann verbreitet Munier an vielen Stellen kaum mehr als wortgewaltige Unwahrheiten.

Eine erste gewagte These lautet, Morus sei ein gnadenloser Ketzerverfolger gewesen, der die Häretiker reihenweise auf die brennenden Scheiterhaufen geschickt habe. Dieser Vorwurf wird in zahlreichen Passagen wiederholt und variiert. $\mathrm{Mu}-$ nier nennt Morus den » Kettenhund « einer inquisitorisch-enthemmten Politik (241). »Es loderten wieder die Scheiterhaufen und der Henker ließ das Fallbeil auf die Häupter unglückseliger Anhänger der Reformation und all derer, die Morus für irregeleitete Abtrünnige vom katholischen Glauben hielt, niedersausen « (239). Muniers Urteil ist nicht nur maßlos überzogen, es geht an den historischen Fakten wohl schlicht vorbei. Richtig ist, dass Morus die Reformation in England in seinen Spätschriften nach Kräften bekämpfte und dass die Ketzerverfolgung dort mit Beginn von Morus' Kanzlerschaft wieder auflebte. Seine Rolle war aber vergleichsweise gering. Die weltliche Macht übernahm zwar die Vollstreckung, doch die Prozesse waren Angelegenheit der Bischöfe und geistlichen Gerichte. Als Laie konnte Morus überhaupt keine Häretiker verurteilen (vgl. Raymond W. Chambers, Thomas Morus, Basel 1947, S. 332). Seine Funktion reduzierte sich darauf, diese in Haft zu halten und Verhöre zu führen. Nach dem Rücktritt von seinen Ämtern schrieb Morus selbst: »Von allen denen, die mir wegen Ketzerei übergeben wurden, hat kein einziger, so wahr mir Gott helfe, einen Schlag oder Streich erhalten, nicht einmal einen Nasenstüber. Alles, was mir meine Amtspflicht auferlegte, war, sie in sicherem Gewahrsam zu halten " (Thomas Morus, The Apolo$g y$ (Complete Works; Bd. 9), New Haven/London 1979, S. 118). Es gibt wenig Grund, die Aussage anzuzweifeln. Kein einziges Ketzer- oder gar Todesurteil existiert, das Morus' Unterschrift trägt. Auch Munier hat ein solches weder gefunden noch gesucht, aber er weiß, dass Morus als Lordkanzler »für solche Urteile verantwortlich war« (241).

Während Morus daher als »Heiliger von zweifelhaftem Ruf « (237) gilt, hält ihn Munier als Vordenker des Kommunismus für "ganz passabel « (243). Dieses Urteil resultiert nun allerdings aus einer völlig naiv-wörtlichen Lesart der »Utopia«. Munier nimmt die Schrift als »verwirklichungsin- 
tendierenden Staatsentwurf « (143) in allen Facetten für bare Münze, obwohl sie an unzähligen Stellen spielerische, experimentelle und oftmals sogar völlig ironisch gemeinte Beschreibungen von Einrichtungen und Sitten enthält. Der historische Morus jedenfalls hat rechtmäßig erworbenen Reichtum mehrfach verteidigt und in einer Spätschrift kommunistisches Gemeineigentum sogar ausdrücklich verworfen (Vgl. Thomas Morus, The Confutation of Tyndale's Answer (Complete Works; Bd. 8,2), New Haven/London 1973, S. 664). Wohl auch deshalb schreibt Munier allen Ernstes: »Kommunist war er nur in jenem schmalen Zeitfenster 1515/16, als er die Utopia verfasste « (243). Selbst wenn dem so wäre, ist das methodische Vorgehen eine Farce. Wenn, dann erlaubt die Person Rückschlüsse auf das literarische Werk, niemals umgekehrt. Übrigens: Die Religion der Utopier ist eine ausgesprochen tolerante und explizit "heidnische" Lehre. Warum der Biograf nicht den Schluss zieht, dass der »Ketzerverfolger « in jener Zeit von einem »heidnischen Fieber « befallen war, das hat er leider für sich behalten.

Noch eine letzte, erstaunliche These Muniers sei hier abschließend erwähnt. Sie lautet auf »Karrierismus « (183). Bei aller vorgegaukelten Bescheidenheit sei Morus in Wahrheit von einer "Ellenbogenmentalität « (71) und "ehrgeizigem Vorwärtskommen « (184) beseelt gewesen. Munier beschreibt Morus als einen Menschen, »der hartnäckig nach oben strebt, zur Not auch opportunistisch den Nacken beugt, um sich bei Förderern einzuschmeicheln und die richtigen Seilschaften $\mathrm{zu}$ finden « (71). Der Biograf scheint dabei kaum noch zu merken, wie sehr er seinem »Gefühl« offenbar mehr traut als allen überlieferten Quellen. Vor allem Erasmus beschreibt in zahllosen Briefen (ebenso wie Morus selbst), die distanzierte, zuweilen unwillige Haltung, wenn es in Morus' Leben darum ging, sich in die Verantwortung politischer Ämter einbinden zu lassen. Auch besonderen Illusionen hat sich Morus dabei offenbar kaum hingegeben. Zu seinem Schwiegersohn William Roper bemerkte Morus einmal über seine Gunst bei König Heinrich VIII.: »Seine Gnaden ist mir wirklich ein sehr gütiger Herr (...). Wie dem auch sei, Sohn Roper, das kann ich dir sagen, ich habe keinen Grund darauf stolz zu sein; denn wenn ihm mein Kopf ein Schloss in Frankreich gewänne (...), würde er unweigerlich fallen " (Roper, aaO., S. 28). Nichts war Morus mehr zuwider als öffentlich zur
Schau gestellter Prunk. Selbst als Lordkanzler hat Morus in seiner Kirche in Chelsea noch den Chorrock angelegt, um zu ministrieren (vgl. Peter Berglar, Die Stunde des Thomas Morus. Einer gegen die Macht, 3. Aufl., Olten/Freiburg 1981, S. 88).

Munier geriert sich, als hätte er als Erster endlich den wahren Morus durchschaut. Unklar aber bleibt beständig, woher er seine Kenntnis des historischen Morus eigentlich nimmt. Die zahlreichen Fehlurteile haben ihre Ursache hauptsächlich in der beachtlichen Souveränität, mit der Munier die Lücken seiner Argumentation durch Berge von Mutmaßungen und Unterstellungen füllt. Angesichts seines Vorhabens ist indes der Verdacht nicht weniger groß, dass er die Quellen auch bei besserem Wissen kaum zur Kenntnis genommen hätte.

Von ganz anderer Seriosität ist dagegen Ribhegges Erasmus-Beschreibung. Rund ein Viertel des Buches umfasst der Anmerkungs- und Literaturapparat (211-272). Das ist nun nicht schlechthin Beleg für Gelehrsamkeit, zumindest aber für Ernsthaftigkeit. Ribhegge ist ein ausgewiesener Experte, bei dem sich das Spektakel schon aufgrund der Detailtreue verbietet. Er bedient sich einer einfachen, unprätentiösen Sprache; gelegentlich hätte man sich den einen oder anderen Spannungsbogen mehr gewünscht. So gesehen ist $\mathrm{Mu}-$ nier - das muss man seiner Darstellung lassen - der eindeutig bessere Stilist von beiden. Ribhegge überzeugt gleichwohl mit breiter und solider Faktenkenntnis.

Die politische Karriere des Morus, das wird in Ribhegges Buch rasch deutlich, ist der wohl größte Unterschied zwischen beiden Humanisten. Erasmus (1467-1536) hat sich zeitlebens allen Angeboten und Anfechtungen weltlicher und geistiger Ämter verweigert. Noch in einem seiner letzten Briefe, einem Schreiben an den Krakauer Bischof Petri Tomicki, berichtet Erasmus vom Angebot Papst Paul III., ihn zum Kardinal zu ernennen. Seine Ablehnung kommentierte Erasmus mit den ironischen Worten: "Soll ich armes kleines Lebewesen, das sozusagen nur noch einen Tag zu leben hat, jetzt in den Wettkampf gegen die Müßiggänger, die Gewalttäter und die Reichen eintreten, nur um reich zu sterben? « Er werde nicht wie ein Ochse sein Joch freiwillig annehmen. Tomicki sah die Angelegenheit übrigens völlig anders. In seinem Antwortschreiben bemühte er zahllose Gründe, um Erasmus doch noch zur Übernahme der Kar- 
dinalswürde zu überreden. Der Brief hat Erasmus nicht mehr erreicht. Er war bereits am 12. Juli 1536 in Basel gestorben. Mit dieser exemplarischen Episode (207-209) endet Ribhegges Buch.

Viele alte, tief eingelagerte Gefühle des Misstrauens sprechen aus den ablehnenden Zeilen des Rotterdamers. Erasmus' Beziehung zur katholischen Kirche ist seit seiner Jugend von vielfachen Ambivalenzen geprägt. Der uneheliche Sohn des Goudaer Priesters Rotger Gerard und seiner Haushälterin, der bereits in jungen Jahre beide Elternteile verlor, trat mit 18 oder 19 Jahren zwar als Novize in das Kloster der Augustinerchorherren in Steyn ein und wurde 1492 zum Priester geweiht. Vor allem das rigide asketische Regime im Pariser Collège Montaigu, wo Erasmus ab 1495 Theologie studierte und im Frühjahr 1496 einen Zusammenbruch erlitt, entfremdeten ihm das Kosterleben aber zusehends (11-27). Im Jahr 1517 wurde er, inzwischen Doktor der Theologie, durch päpstlichen Dispens endgültig von seinem Ordensgelübde entbunden (83-84). Anders als Morus machte sich Erasmus auch nie zu einem Parteigänger in den kirchenpolitischen Auseinandersetzungen seiner Zeit. So sehr er den Ämterkauf und die Privilegien der alten Kirchenfürsten anprangerte, so sehr er die Schwerfälligkeit und Absurditäten der spätscholastischen Theologie (nicht zuletzt im "Lob der Torheit «) verspottete, so wenig Sympathie hegte er für Luther, seine Anhänger und die Reformation (103-137).

$\mathrm{Zu}$ Weltruhm gelangte Erasmus letztlich allein mit seinen Schriften, die an Gelehrsamkeit und Formulierungskunst ihresgleichen suchen, und durch ein einzigartiges Netzwerk an Korrespondenzen, das er mit fast allen europäischen Gelehrten und Höfen unterhielt. Überall suchte man seinen Kontakt und man hütete Erasmus' Briefe eitel wie kleine Schätze. Percy Stafford Allen (nicht "John Percy Allen «, wie Ribhegge schreibt, 9) begann 1906 mit der Veröffentlichung von Erasmus' Schriftwechsel in der Oxforder Ausgabe »Opus Epistolarum Des. Erasmi Roterodami« (vgl. Erasmus, aaO.). Es wurde ein Lebenswerk. Der zwölfte und letzte Band erschien 1958. Insgesamt umfasst die Edition mehr als 3000 Briefe von und an Erasmus. Die Ausgabe liefert heute ein kaum zu übertreffendes Bild der Zustände und Konflikte im Zeitalter der Renaissance.

Das ironisch-spöttische Gemüt war Morus wie Erasmus aber gleichermaßen eigen. Legendär ist
Erasmus' Szene, in der er Papst Julius II. nach dessen Tod in Kriegsrüstung vor der Himmelstür ein Streitgespräch mit Petrus führen lässt, weil ihm dieser den Zutritt verweigert (vgl. Erasmus von Rotterdam, Julius vor der verschlossenen Himmelstür, ein Dialog, in: ders., Ausgewählte Schriften, hrsg. v. Werner Welzig, Bd. 5, Darmstadt 1968, S. 6-109). In solchen Passagen wusste er sich mit Morus einig. Schon Morus widmete in seiner »Utopia« der kriegerischen und vertragsbrüchigen Kirchenstaatspolitik unter Julius II. eine bitterböse Satire (vgl. Thomas Morus, Utopia, in: Der utopische Staat, hrsg. v. Klaus Heinisch, Reinbek bei Hamburg 1996, S. 86). Seine Widmung an Morus im »Lob der Torheit «, das Erasmus bei Morus in London niederschrieb und das dem Freund wohl nicht nur wegen der Namensähnlichkeit (griech. mori $=$ Torheit) zugeeignet ist, beschließt Erasmus mit der spielerischen Aufforderung: »Du bist ein so vorzüglicher Anwalt, dass du selbst eine bedenkliche Sache erfolgreich vertreten kannst. Leb wohl, beredter und gewandter Freund Morus, und mach die Sache der Moria zu deiner eigenen " (Erasmus von Rotterdam, Das Lob der Torbeit. Encomium Moriae. Hrsg. von Anton J. Gail, Stuttgart 1985, S. 6). Aus dem Spiel wurde Ernst. Erasmus' Schrift rief zum Teil erbitterte Kritiker auf den Plan. Unter anderem forderte der Löwener Theologe Martin Dorp, Erasmus sollte als Wiedergutmachung ein »Lob der Vernunft «schreiben. In einem langen Brief verteidigte Morus später nicht nur Erasmus und dessen Schrift. Nur wenige Monate später entstand auch seine »Utopia «. Dort wird die Vernunft wieder und wieder als das eigentliche und beherrschende Fundament aller Sitten und Institutionen gerühmt, zuweilen ernst, zuweilen ironisch. Beweisen lässt es sich nicht, aber der Verdacht ist gewaltig, dass sich Morus nicht nur als »Anwalt « des Erasmus verwendete, sondern auch, dass er mit seiner »Utopia « die eigentlich dem Freund zugedachte Aufgabe übernommen hat. Das Verhältnis von Erasmus und Morus jedenfalls, das macht auch die Lektüre der beiden Biografien deutlich, ist für die Interpretation einer Vielzahl ihrer Werke noch keineswegs endgültig erschlossen.

Thomas Schölderle 
Martin THEIN: Wettlauf mit dem Zeitgeist - Der Neonazismus im Wandel. Eine Feldstudie, Göttingen: Cuvillier Verlag 2009, 467 S., kart., 39 EUR.

Die Zahl der Aktivisten der Neonazi-Szene hat sich in den letzten Jahren verdoppelt: von über 2.000 Anfang der 1990er Jahre auf gegenwärtig über 4.000. Die Ursachen für diese Entwicklung will der Politikwissenschaftler Martin Thein in seiner Arbeit Wettlauf mit dem Zeitgeist - Der Neonazismus im Wandel. Eine Feldstudie herausarbeiten. Es soll darin um die »Ursachen, Erfolgsbedingungen und Charakteristiken « und die daraus »resultierende(n) Attraktivitätsmomente für ein solches Denken und Handeln « (S. 3) gehen. Hierbei will der Autor - bei Ausblendung des gesamtgesellschaftlichen Kontextes - nur die szene-interne Entwicklung betrachten, ebendort die ideologischen, organisatorischen und strategischen Veränderungen ins Visier nehmen und einen Vergleich der Neonazi-Szene der 1970er und 1980er Jahre mit der der Gegenwart vornehmen. Primäre Datenbasis dafür sind 34 Tiefeninterviews mit noch aktiven und ehemaligen Angehörigen dieses Lagers des Rechtsextremismus, wozu auch ein Großteil der Führungsriege des Neonazismus gehörte.

Nach der Einleitung mit Ausführungen zu Erkenntnisinteresse und Fragestellung, Forschungsstand und Methode finden sich Ausführungen zu den zentralen Arbeitsbegriffen »Neonazismus « und »Rechtsextremismus « sowie zur historischen Entwicklung des Neonazismus in Deutschland. Dem folgen die drei Hauptteile der Arbeit, worin nach den organisatorischen, ideologischen und strategischen Veränderungen in diesem Teil des Rechtsextremismus jeweils für die 1970er und 1980er sowie die 1990er und 2000er Jahre gefragt wird. Hierbei geht es um so unterschiedliche Aspekte wie das traditionelle Organisationsmodell und die »Kameradschafts«-Strukturen, die ideologische Neuorientierung, die strategische Binnenfixierung und die Neuausrichtung als gesellschaftliche Akteure. Der bilanzierende Schlussteil unterscheidet dann idealtypisch zwei Formen der Angehörigen dieses politischen Spektrums: den »traditionellen (westdeutschen) Neonazi« (S. 322) und den »Neonazi neuen Typs« (S. 325).

Dementsprechend formuliert Thein als zentrales Ergebnis seiner Forschungsarbeit »das Aufzeigen der Existenz eines neune Typs an Neonazi in
Ostdeutschland, dessen Charakteristika sich elementar von dem... klassischen westdeutschen Akteur unterscheiden « (S. 325). Diesen definiert er wie folgt: »Unter,Modernen Neonazis verstebt man Rechtsextremisten, die sich vorwiegend in regionalen Kleingruppierungen organisieren und aus strategischen Gründen ibre vormals primär nationalistische Orientierung durch sozialpolitische, $k a-$ pitalismuskritische und lokalbezogene Themeninhalte erweitert haben «(S. 326, kursiv im Original). In diesem Kontext wird auch auf den szeneinternen ideologischen Wandel hingewiesen, habe sich doch insbesondere in Ostdeutschland ein »differenziertes Verhältnis zum historischen Nationalsozialismus " herausgebildet. Als entscheidende Ursache für den »Attraktivitätszuwachs « wird die »binnenorganisatorische Neuausrichtung in Form des Kameradschaftsmodells" (S. 329) ausgemacht.

Die Arbeit von Thein beeindruckt durch das methodische Vorgehen: Erstmals liegt hier eine wissenschaftliche Studie vor, welche auf Basis von Tiefeninterviews mit zahlreichen führenden Neonazi-Aktivisten der Vergangenheit und Gegenwart erstellt wurde. Deren systematische Auswertung wird im Anhang mit den Ausführungen zur Methode gut begründet. Der Autor nimmt darüber hinaus auch eine überzeugende Strukturierung bei der Präsentation seiner Ergebnisse vor, wobei eine ideologische, organisatorische und strategische Ebene unterschieden werden. Gleichzeitig verkoppelt er diese Differenzierung der Untersuchungsfelder mit dem Vergleich des früheren und gegenwärtigen Neonazismus. Hierbei gelingt es überzeugend, neuere Entwicklungen in diesem Teil des Rechtsextremismus herauszuarbeiten. Thein arbeitet auch in der Gesamtanalyse die wichtigsten Bedingungsfaktoren für den »Attraktivitätszuwachs « der Szene auf und gewichtet diese klar hinsichtlich ihrer Bedeutung (entscheidender Faktor: Organisationsstruktur).

Diesen Vorzügen stehen aber auch kleinere Schwächen gegenüber: Die zentrale Fragestellung hätte klarer und systematischer - auch und gerade hinsichtlich der Gewichtung von Hauptfragestellung und Unterfragen - entwickelt werden müssen. Bei der Auswertung der Interviews geht der Autor zu wenig auf die subjektive Interessenlage der Befragten ein, was sich an der häufig kritikarmen Rezeption ihrer Aussagen zeigt. Zwar gibt es einen »neuen Typ « des Neonazis, der sich insbesondere in den ostdeutschen Ländern herausgebil- 
det hat. Aber auch der »alte Typ« kannte durchaus »»sozialpolitische, kapitalismuskritische... Themeninhalte«(S. 329), womit dies kein trennscharfes Kriterium sein kann. Gleichwohl handelt es sich bei Theins Arbeit in der Gesamtschau um eine bedeutende Forschungsleistung, die in der systematischen Auswertung der Tiefeninterview mit wichtigen Vertretern der Neonazi-Szene und der problemorientierten Analyse bezüglich der ideologischen, organisatorischen und strategischen Entwicklung auszumachen ist.

Armin Pfabl-Tranghber

\section{Ein zeitloses Märchen.}

Der Triumph der Gerechtigkeit

Wer mit seinen Nachbarn in Frieden lebt, kennt nur seine Rechte nicht

Ein zeitloses Märchen. Mit

Zeichnungen von Imma Setz

Von Rudolf Gerhardt

2009, 115 S., brosch., 19,- $€$,

ISBN 978-3-8329-5302-7

Rudolf Gerhardt, Jurist und Journalist, beschreibt mit einem „gewissen Lächeln“ den

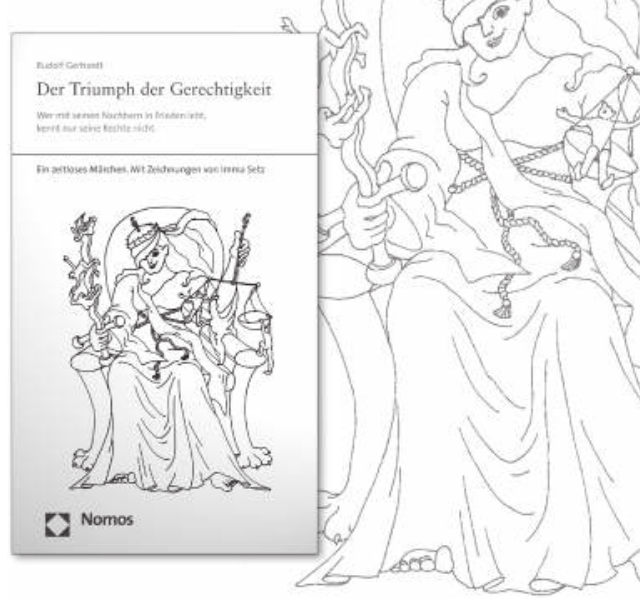

Einbruch des Rechts in Person eines zugezogenen jungen Anwalts in die friedliche Idylle eines Schwarzwalddorfes, wo die Bewohner bis dahin nicht auf die Idee gekommen waren, sich um Recht und Gerechtigkeit zu streiten. Dass der junge Anwalt auf den verschlungenen Rechtswegen, auf die er seine Mandanten lockte, schließlich selbst ins Straucheln gerät, mag als Triumph der Gerechtigkeit angesehen werden. Am Gerechtesten aber, so Gerhardts Fazit, geht es noch immer dort zu, wo sich die Menschen, gleichsam im Zustand der Unschuld, ums Recht-haben oder Recht-bekommen nicht scheren.

Gisela Friedrichsen, DER SPIEGEL

Bitte bestellen Sie im Buchhandel oder versandkostenfrei unter $\bullet$ www.nomos-shop.de

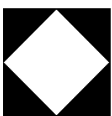

